\title{
Diblock/Triblock Structural Transition and Sol-Gel Transition of Peptide/PEG Diblock Copolymer Having a Terminal Terpyridine Group Induced by Complexation with Metal Ion
}

\author{
Satoshi Tanimoto, Yasushi Nakamura, Hitoshi Yamaoka, and Yoshitsugu Hirokawa \\ Department of Materials Science, The University of Shiga Prefecture, Hassaka, Hikone 522-8533, Japan \\ Correspondence should be addressed to Satoshi Tanimoto, tanimoto@mat.usp.ac.jp
}

Received 26 January 2010; Accepted 21 June 2010

Academic Editor: Harald W. Ade

Copyright (C) 2010 Satoshi Tanimoto et al. This is an open access article distributed under the Creative Commons Attribution License, which permits unrestricted use, distribution, and reproduction in any medium, provided the original work is properly cited.

Terpyridine-polyethyleneglycol-block-polyleucine block copolymer (tpy-PEG-PLeu) was synthesized by a ring-opening polymerization of L-leucine $\mathrm{N}$-carboxyanhydride. The copolymer complexed with $\mathrm{Fe}^{2+}$ ion and its aqueous solution showed a purple color as a result of the complexation. This complexation caused the diblock/triblock structural transition of the copolymer. The change of the aggregation behavior caused by the structural transition was observed by a dynamic light scattering apparatus. The diblock tpy-PEG-PLeu copolymer formed a micelle in the aqueous solution. On the other hand, the triblock-type copolymer, after the complexation, formed the micelle structures and huge aggregates, which is considered to be a network structure. The complexation of the diblock tpy-PEG-PLeu copolymer with Fe ion is consequently considered to be a trigger of the gelation.

\section{Introduction}

The chloroform solution of the ABA-type triblock copolymer which consists of a polyleucine (PLeu) chain as the A segment and a polyethylene glycol (PEG) as the B segment has been reported to show a thermoresponsive sol-gel transition [1]. This sol-gel transition was not observed on the solution system of AB-type block copolymer having the same components. These results were attributed to the difference in the aggregation behavior between the $\mathrm{AB}$ type and the ABA-type block copolymers. Since the two polyleucine segments of the triblock copolymer behave as the junction point due to the hydrophobic interaction to string the many copolymers together, the ABA-type triblock copolymer in the solution can form the network structure, resulting in the formation of the gel. On the other hand, the AB-type diblock copolymer could not form the gel like the ABA-type [2].

On the basis of the facts mentioned above, we would like to propose the novel sol-gel transition system made up of the AB-type diblock copolymer as a basic material. In this study, the terminal functionalized $\mathrm{AB}$-type diblock copolymer consisting of PEG and polyleucine segments was synthesized. A terpyridine, which was known to make a complex with metal ion [3-6], was employed as a terminal functional group. The terminal functionalized $\mathrm{AB}-$ type diblock copolymer consisting of PEG and polyleucine segments and having the terpyridine group (tpy) at the end of PEG is, hereafter, referred to as tpy-PEG-PLeu.

When the divalent metal ion is added to the solution of the tpy-PEG-PLeu, the metal ion should behave as the terminal connector of the diblock copolymer and make the tpy-PEG-PLeu into the ABA-type triblock copolymer. Therefore, the addition of the divalent metal ion could be expected to induce the diblock/triblock structural transition followed by the network formation of gel. The aggregation behavior of the block copolymer was investigated by a dynamic light scattering (DLS) apparatus.

\section{Experimental}

2.1. Materials. $4^{\prime}$-chloro-2, $2^{\prime}: 6^{\prime}, 2^{\prime \prime}$-terpyridine (tpy) was purchased from Aldrich and used as received. $\alpha$ aminopropyl- $\omega$-hydroxypolyoxyethylene (HO-PEG-NH ${ }_{2}$ ) 


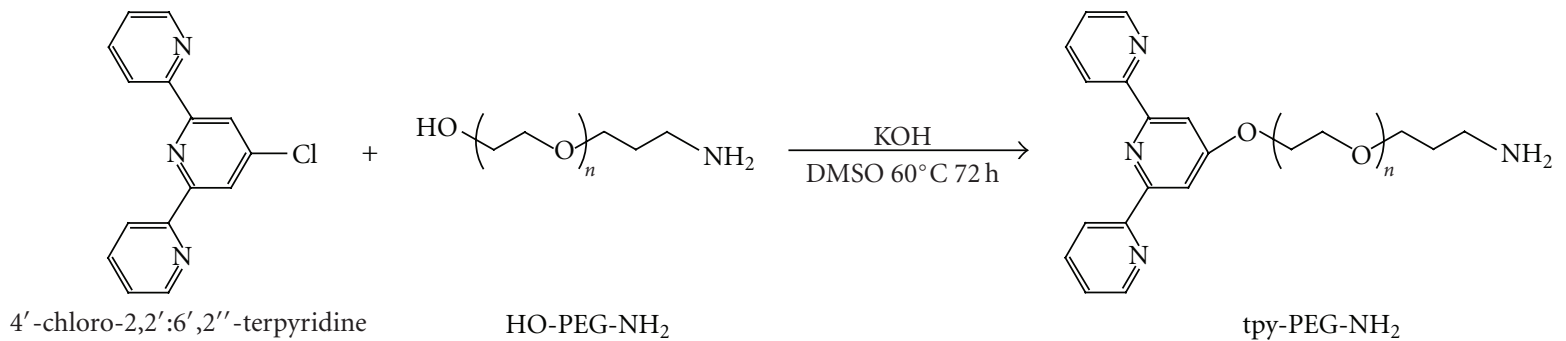

SCHEME 1: Synthesis procedure of macroinitiator, tpy-PEG-NH $\mathrm{NH}_{2}$.

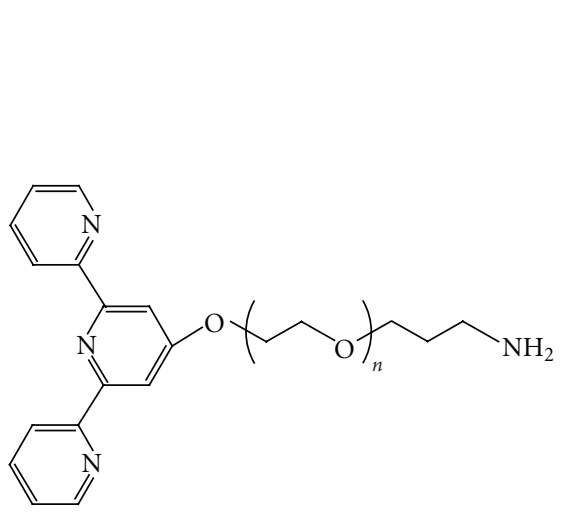

tpy-PEG-NH

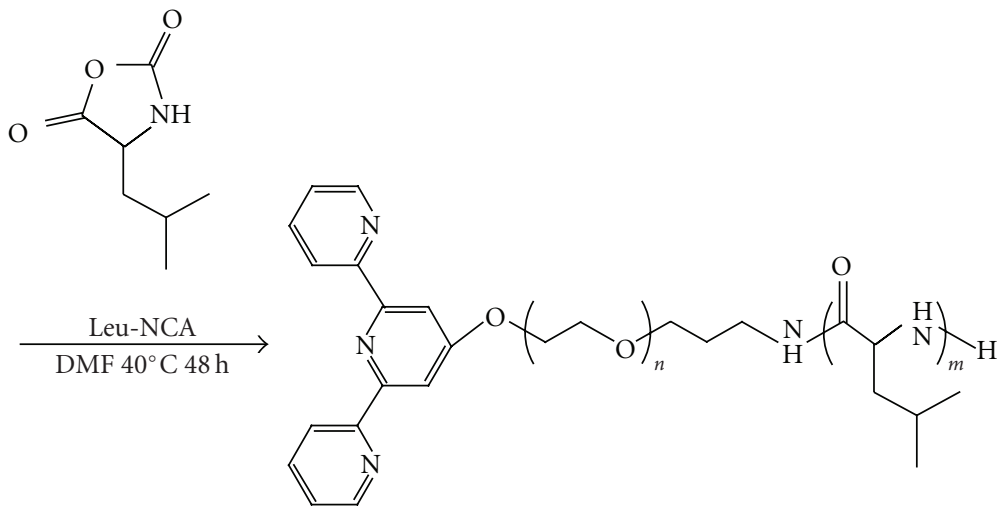

tpy-PEG-P(Leu)

Scheme 2: Copolymerization of tpy-PEG-PLeu.

(SUNBRIGHT HOPA-2000) was purchased from NOF Corp. Ltd. and used after reprecipitation into diethyl ether from chloroform solution. L-leucine was purchased from Wako Pure Chemicals Ltd., Tokyo, Japan, and it was used without further purification. Triphosgene and sodium sulfate were purchased from Nacalai Tesque, Osaka, Japan and used as received. All solvents used in the synthesis procedures were purchased from Wako Pure Chemicals Ltd., Tokyo, Japan, and used after appropriate desiccating treatment.

2.2. Characterization Methods. Characterization of the copolymers was performed by gel permeation chromatograph (GPC) (Series-10A, Shimadzu Corporation) with polystyrene columns (Polymer Laboratory, Mixed-C and Mixed-E) and by a nuclear magnetic resonance (NMR) (Lambda-400, JEOL Ltd.). Diameter of the copolymer micelle was measured by a dynamic light scattering (DLS) apparatus (DLS-7000, Otsuka Electronics, Japan). Spectroscopic analysis was carried out with Fourier transform Infrared spectrometer (FT-IR430, Jasco, Japan), and UVvisible spectrometer (U-3000, Hitachi, Japan).

2.3. Synthesis of tpy-PEG-PLeu. PEG, having a terpyridine group and an amino group on each end (tpy-PEG- $\mathrm{NH}_{2}$ ), was synthesized under nitrogen atmosphere by the procedures as shown in Scheme 1. HO-PEG-NH $2(3.0 \mathrm{mmol})$ was dissolved in DMSO $(100 \mathrm{~mL})$, and $\mathrm{KOH}(15 \mathrm{mmol})$ was added to the solution. The mixture was stirred for one hour at $60^{\circ} \mathrm{C}$.
After the addition of tpy $(3.0 \mathrm{mmol})$, the solution was further stirred for 72 hours at $60^{\circ} \mathrm{C}$ and then filtered off the excess of $\mathrm{KOH}$. After evaporation of DMSO, the product was dissolved by chloroform $(30 \mathrm{~mL})$ and desiccated on sodium sulfate, then the residual tpy was removed by filtration. tpy-PEG$\mathrm{NH}_{2}$ was obtained as a yellowish oily product, after removal of chloroform.

L-leucine was converted into $\mathrm{N}$-carboxyanhydride (LeuNCA) as a monomer by Fuchs-Farthing method [7]. The procedure was described in detail in our previous paper [1]. Molar ratio of L-leucine to triphosgene was $3: 2$, and the reaction was carried out in THF for one hour at $40^{\circ} \mathrm{C}$. The reaction was performed until the solution became transparent. The product was purified by three-time recrystallization with $\mathrm{THF} / \mathrm{h}$ exane and obtained as white powder after freeze-drying.

Diblock copolymer consisting of PEG segment terminated by tpy group and polyleucine segment (tpyPEG-PLeu) was synthesized by the procedure as shown in Scheme 2. The whole process was carried out under nitrogen atmosphere. The tpy-PEG- $\mathrm{NH}_{2}$ was added to LeuNCA/DMF solution as an initiator. The polymerization was conducted for 48 hours at $40^{\circ} \mathrm{C}$. After the polymerization, the product was purified by precipitation with diethyl ether. The precipitate was freeze-dried to obtain a white powdery product, which is tpy-PEG-PLeu copolymer.

2.4. Metal Ion Complexation of tpy-PEG-PLeu. $0.22 \mathrm{~g}$ of tpyPEG-PLeu dissolved in $30 \mathrm{~mL}$ of chloroform and $1.0 \mathrm{mg}$ of 


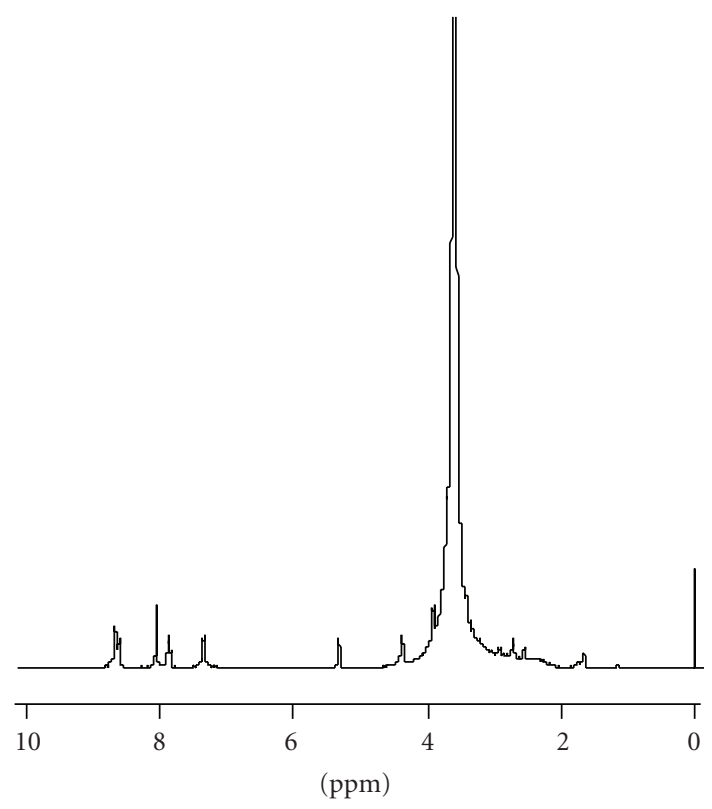

FIGURE 1: NMR spectrum of tpy-PEG-NH $\mathrm{NH}_{2}$. Solvent: chloroform-d, temperature: $20^{\circ} \mathrm{C}$.

$\mathrm{FeCl}_{2}$ dissolved in $20 \mathrm{~mL}$ of methanol was mixed and kept for 6 hours at $40^{\circ} \mathrm{C}$ to make a complex of terpyridine group with Fe ion [8]. The solvent was evaporated and the product was finally freeze-dried from benzene. The tpy-PEG-PLeu with Fe complex was thus obtained.

2.5. Evaluation of tpy-PEG-PLeu Aggregation. The aggregate size of tpy-PEG-polyleucine was measured in chloroform at $20^{\circ} \mathrm{C}$ by DLS equipped with He-Ne laser. The block copolymers were of diblock type, tpy-PEG-PLeu, and triblock type, and the concentration of the block copolymer was $1.0 \mathrm{~g} / \mathrm{L}$ for both types.

\section{Results and Discussion}

3.1. Characterization of tpy-PEG-PLeu. The macroinitiator, tpy-PEG-NH $\mathrm{N}_{2}$, obtained by the reactions, as shown in the Scheme 1, was characterized by NMR spectroscopy. Figure 1 shows NMR spectra of the tpy-PEG-NH $\mathrm{N}_{2}$. The fraction of the polymer having the tpy unit was calculated from the integrated area ratio of the signals assigned to the tpy protons found at 7.0-9.0 ppm to the signal assigned to PEG protons at $3.0-4.2 \mathrm{ppm}$. The number-averaged molecular weight and the polydispersity index of the tpy-PEG- $\mathrm{NH}_{2}$ was found to be 1882 and 1.01, respectively, by means of GPC measurements.

The monomer, leu-NCA, was polymerized with tpyPEG- $\mathrm{NH}_{2}$ as a macroinitiator. After 48 hours from the addition of the monomer to the tpy-PEG- $\mathrm{NH}_{2}$, IR absorption band assigned to NCA-ring structure disappeared. The reaction mixture was poured drop by drop into diethyl ether, and the white precipitate was recovered by filtration. The yield throughout the total synthesis procedures was about $60 \%$.

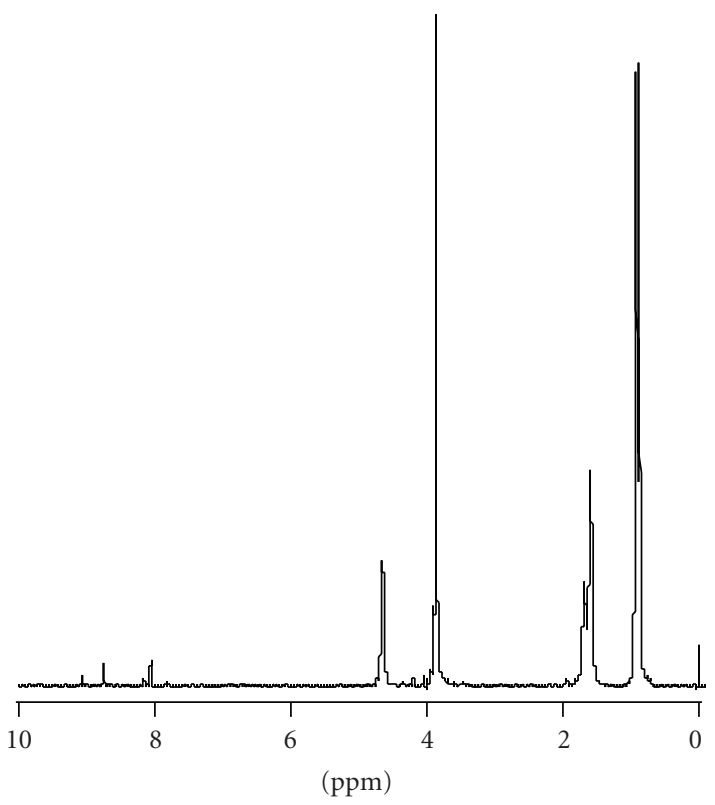

FIGURE 2: NMR spectrum of the final product, tpy-PEG-PLeu. The polymerization degree of the peptide segment, that is, the molecular weight, and the fraction of the polymer chain having the tpy unit at the end in Table 1 were obtained by NMR measurements. The polydispersity index, $\mathrm{Mw} / \mathrm{Mn}$, was obtained by GPC measurements.

TABle 1: Characterization of tpy-PEG-PLeu.

\begin{tabular}{|c|c|c|c|c|}
\hline & PEG : PLeu ${ }^{(1)}$ & M.W. ${ }^{(1)}$ & $\mathrm{Mw} / \mathrm{Mn}^{(2)}$ & {$[\mathrm{tpy}] /\left[\right.$ polymer] $(\%)^{(1)}$} \\
\hline EG45L14 & $45: 14$ & 3980 & 1.38 & 89 \\
\hline EG45L24 & $45: 24$ & 5280 & 1.43 & 92 \\
\hline EG45L41 & $45: 41$ & 7490 & 1.51 & 91 \\
\hline
\end{tabular}

${ }^{(1)}$ Calculated from ${ }^{1} \mathrm{H}$ NMR spectra. ${ }^{(2)}$ Measured by GPC.

The final products were characterized by NMR and GPC. Figure 2 shows the proton NMR spectrum of the product, tpy-PEG-PLeu. In Figure 2, the signals observed at 7.7-9.2 and 3.5-4.1 ppm were assigned to terpyridine group and PEG chain, respectively. The signals observed at $0.7-2.0 \mathrm{ppm}$ and $4.7 \mathrm{ppm}$ were assigned to the peptide segment. GPC curve of the tpy-PEG-PLeu showed only the monodisperse peak but not other peaks, which might suggest that the amount of impurities was inevitably small. The product was thus confirmed to be the tpy-PEG-PLeu copolymer.

The characteristics of the copolymer were summarized in Table 1. The polymerization degree of the used precursor, HO-PEG- $\mathrm{NH}_{2}$, was fixed to be 45 and that of the peptide segment was found to be varied in the range from 14 to 41 depending on the introduced amount of the monomer, Leu-NCA. The polymerization degree of the peptide chain was thus easily controlled by the amount of the introduced monomer.

3.2. Metal Ion Complexation of tpy-PEG-PLeu. Color change of the tpy-PEG-PLeu solution caused by metal ion complexation was observed and shown in Figure 3. After $\mathrm{Fe}^{2+}$ ion was added to the tpy-PEG-PLeu chloroform solution, 


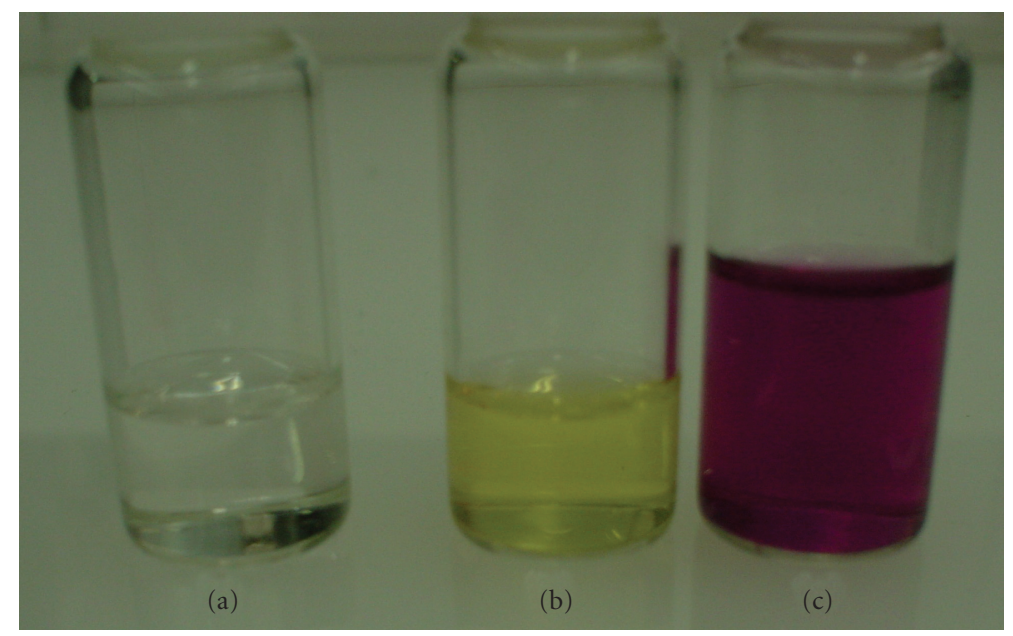

Figure 3: Color change of the tpy-PEG-PLeu solution caused by the complexation with metal ion. (a) Chloroform solution of tpy-PEGPLeu. (b) Methanol solution of $\mathrm{Fe}^{2+}$ ion. (c) Mixture of the solution (a) and (b).

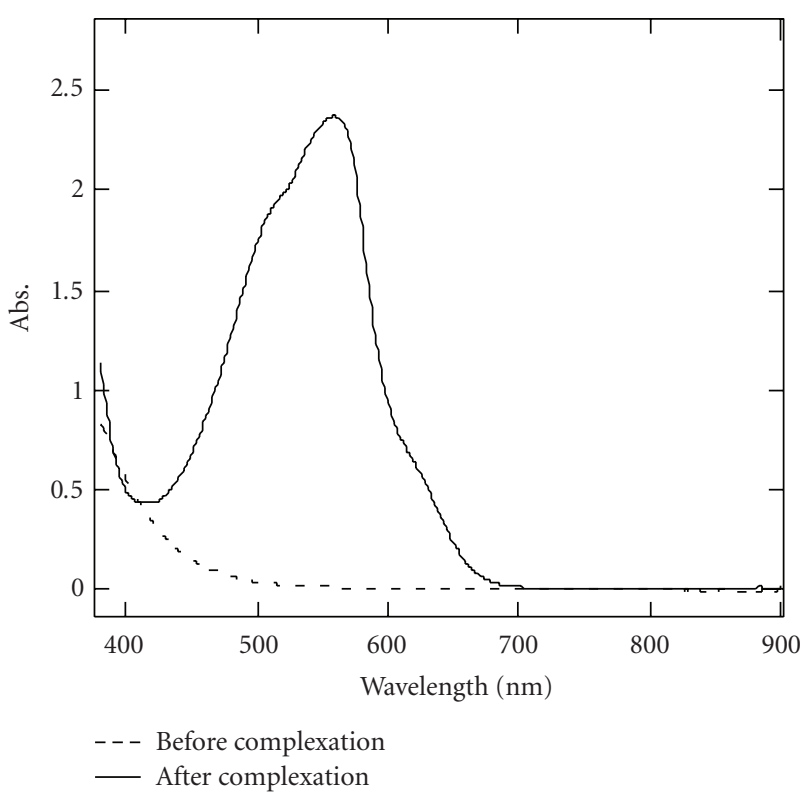

Figure 4: UV-Vis spectra of tpy-PEG-PLeu before and after complexation with $\mathrm{Fe}^{2+}$ ion.

the solution changed its color from transparent colorless to purple as shown in Figure 3(c). UV-vis absorption spectrum of the purple solution was measured and shown in Figure 4. The absorption peak was found around $580 \mathrm{~nm}$ and assigned to the metal ligand charge-transfer complex of the tpy unit with $\mathrm{Fe}^{2+}$ [8]. The metal ion complexation was also confirmed by the change of the molecular weight, where those of the AB-type block copolymer (EG45L14) before and after complexation measured by a static light scattering (SLS) apparatus were 3077 and 7808, respectively. The molecular weight of the copolymer became doubled after the addition of the metal ion. Therefore, these facts indicate that the tpy unit at the end of the diblock copolymer can form the complex with Fe ion, which connects the two diblock copolymers.

3.3. Aggregation Behavior of tpy-PEG-PLeu. Since chloroform is a good solvent of PEG but is a poor solvent of PLeu, the tpy-PEG-PLeu will form the micelle structure having a core region composed of PLeu chains in chloroform. Aggregation behavior of the tpy-PEG-PLeu copolymer in the chloroform solution was investigated by DLS measurement before and after complexation with metal ion. The concentration of the copolymer solution was fixed to be $1.0 \mathrm{~g} / \mathrm{L}$, which was above the critical micelle concentration. Figure 5 shows the size distributions of aggregates formed in the chloroform from the tpy-PEG-PLeu (EG45L24) before and after the complexation with metal ion. Figure 5(a) shows the size distribution of the micelle formed by the tpy-PEGPLeu diblock copolymers themselves. The histogram showed a single-peak profile having $70 \mathrm{~nm}$ as an average diameter. The chain length of the copolymer EG45L24 was estimated to be about $30 \mathrm{~nm}$ as a fully stretched trans-zigzag chain. The size of the micelle was acceptable on the basis of its polymerization degree. These facts indicate that the PEG chain as a corona part of the micelle takes fully stretched form in the chloroform solution. On the other hand, in the case of the triblock copolymer formed by the complexation of tpy-PEG-PLeu with metal ion, the micelles having the same size as that observed before complexation and huge aggregate with the size of over $1000 \mathrm{~nm}$ were observed, as shown in Figure 5(b). This huge aggregate, which is formed from the triblock-type copolymers, is considered to play an important role in the polymer network formation [9].

3.4. Gelation of tpy-PEG-PLeu. The gelation behavior of the $5.0 \mathrm{wt} \%(75 \mathrm{~g} / \mathrm{L})$ chloroform solutions of the copolymer before and after complexation was investigated. The solution of the copolymer having a triblock structure showed a sol-gel transition as the temperature was cooling from room temperature down to $0^{\circ} \mathrm{C}$. This sol-gel transition 


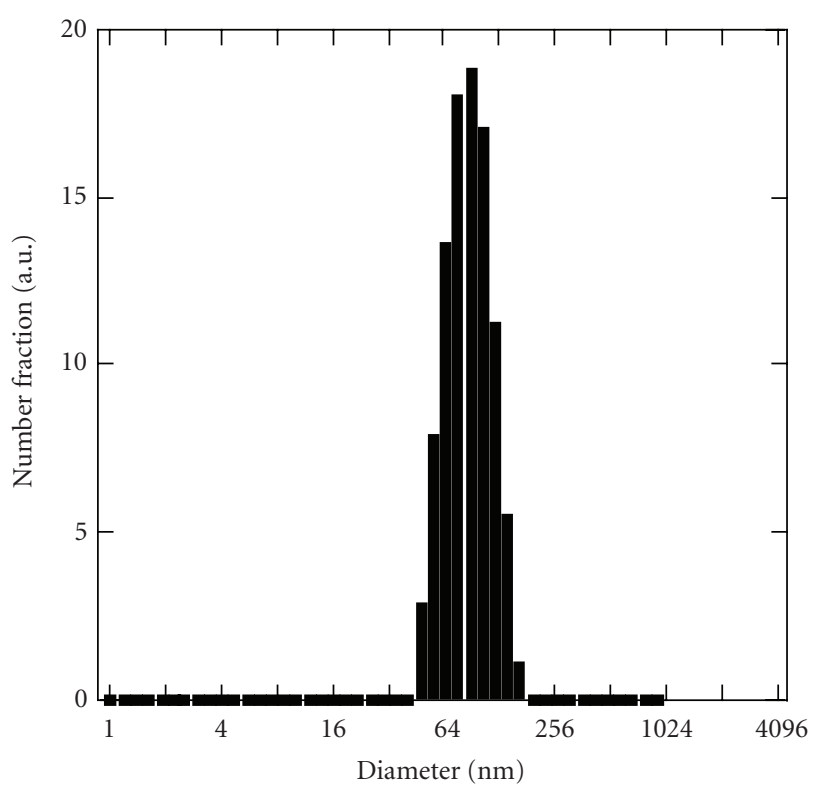

(a)

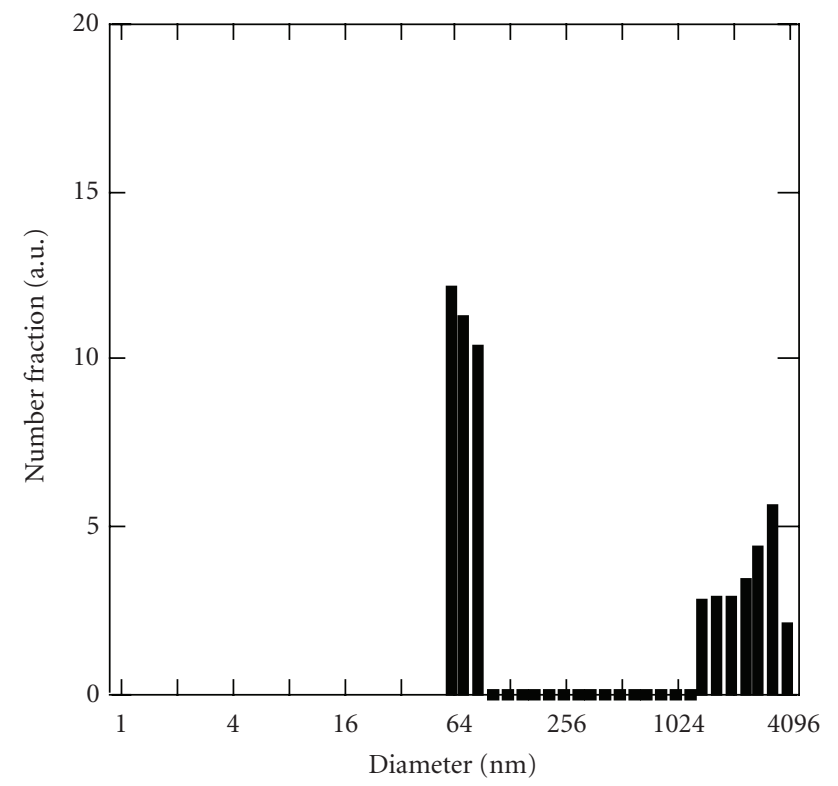

(b)

FIGURE 5: Size distribution of aggregates obtained from (a) diblock-type structure and (b) triblock-type structure. Copolymer: EG45L24, concentration: $1.0 \mathrm{~g} / \mathrm{L}$, temperature: $20^{\circ} \mathrm{C}$.

was a reversible phenomena depending on the temperature. On the other hand, the solution of the diblock copolymer without complexation did not show the sol-gel transition even when the temperature was cooling down below $-50^{\circ} \mathrm{C}$. The transition temperature increased with the increase of the peptide length. In the case of EG45L41 having the longest peptide chain among the used copolymer samples, the transition temperature was found to be $7^{\circ} \mathrm{C}$. In the case of the diblock copolymer before the complexation with metal ion, the solution system did not show the sol-gel transition, since there was no bridging molecule, which is necessary to form network structures. Therefore, the huge aggregation indicated in Figure 5(b) was considered to have the same structure as the junction point existing in the gel phase of the thick solution of the triblock-type copolymer.

\section{Conclusion}

tpy-PEG-PLeu, which had a terminal terpyridine group, was synthesized by a ring-opening polymerization of Leu-NCA initiated by the macroinitiator having amino group. The diblock copolymer complexed with $\mathrm{Fe}^{2+}$ ion and its aqueous solution showed a purple color. This complexation caused the diblock/triblock structural transition of the copolymer. The change of the aggregation behavior caused by the structural transition was estimated by means of DLS. The diblock tpy-PEG-PLeu formed a micelle in the aqueous solution. On the other hand, the triblock-type copolymer after the complexation with metal ion showed the micelle structures and the huge aggregates, which are considered to form the network structures. Eventually, the complexation of the diblock copolymer, tpy-PEG-PLeu, with $\mathrm{Fe}$ ion, which is considered to be a trigger of gelation, caused the diblock/triblock structural transition, which induced the network formation of the triblock polymer, resulting in the formation of the gel.

\section{References}

[1] S. Tanimoto, N. Yagi, and H. Yamaoka, "Application of thermosensitive peptide copolymer gels to removal of endocrine disruptor," Research Letters in Materials Science, vol. 2009, Article ID 597308, 4 pages, 2009.

[2] S. Tanaka, A. Ogura, T. Kaneko, Y. Murata, and M. Akashi, "Precise synthesis of ABA triblock copolymers comprised of poly(ethylene oxide) and poly( $\beta$-benzyl-l-aspartate): a hierarchical structure inducing excellent elasticity," Macromolecules, vol. 37, no. 4, pp. 1370-1377, 2004.

[3] S. Schmatloch, A. M. J. Van Den Berg, A. S. Alexeev, H. Hofmeier, and U. S. Schubert, "Soluble high-molecular-mass poly(ethylene oxide)s via self-organization," Macromolecules, vol. 36, no. 26, pp. 9943-9949, 2003.

[4] U. S. Schubert, C. Eschbaumer, P. Andres et al., " $2,2^{\prime}: 6^{\prime}, 2^{\prime \prime}$ terpyridine metal complexes as building blocks for extended functional metallo-supramolecular assemblies and polymers," Synthetic Metals, vol. 121, no. 1-3, pp. 1249-1252, 2001.

[5] J. S. Choi, C. W. Kang, K. Jung, J. W. Yang, Y.-G. Kim, and H. Han, "Synthesis of DNA triangles with vertexes of bis(terpyridine)iron(II) complexes," Journal of the American Chemical Society, vol. 126, no. 28, pp. 8606-8607, 2004.

[6] G. W. M. Vandermeulen, C. Tziatzios, D. Schubert et al., "Metal ion assisted folding and supramolecular organization of a De Novo designed metalloprotein," Australian Journal of Chemistry, vol. 57, no. 1, pp. 33-39, 2004.

[7] A. Harada and K. Kataoka, "Formation of polyion complex micelles in an aqueous milieu from a pair of oppositely-charged block copolymers with poly(ethylene glycol) segments," Macromolecules, vol. 28, no. 15, pp. 5294-5299, 1995.

[8] B. G. G. Lohmeijer and U. S. Schubert, "Water-soluble building blocks for terpyridine-containing supramolecular polymers: synthesis, complexation, and $\mathrm{pH}$ stability studies of poly(ethylene oxide) moieties," Macromolecular Chemistry and Physics, vol. 204, no. 8, pp. 1072-1078, 2003. 
[9] M. Kasuya, K. Inomata, and E. Nakanishi, "Helix-coil transition and association behavior of both-end hydrophobicallymodified poly[ $\mathrm{N}^{5}$-(2-hydroxyethyl) L-glutamine]," Polymer Preprints, Japan, vol. 54, no. 1, p. 737, 2005. 

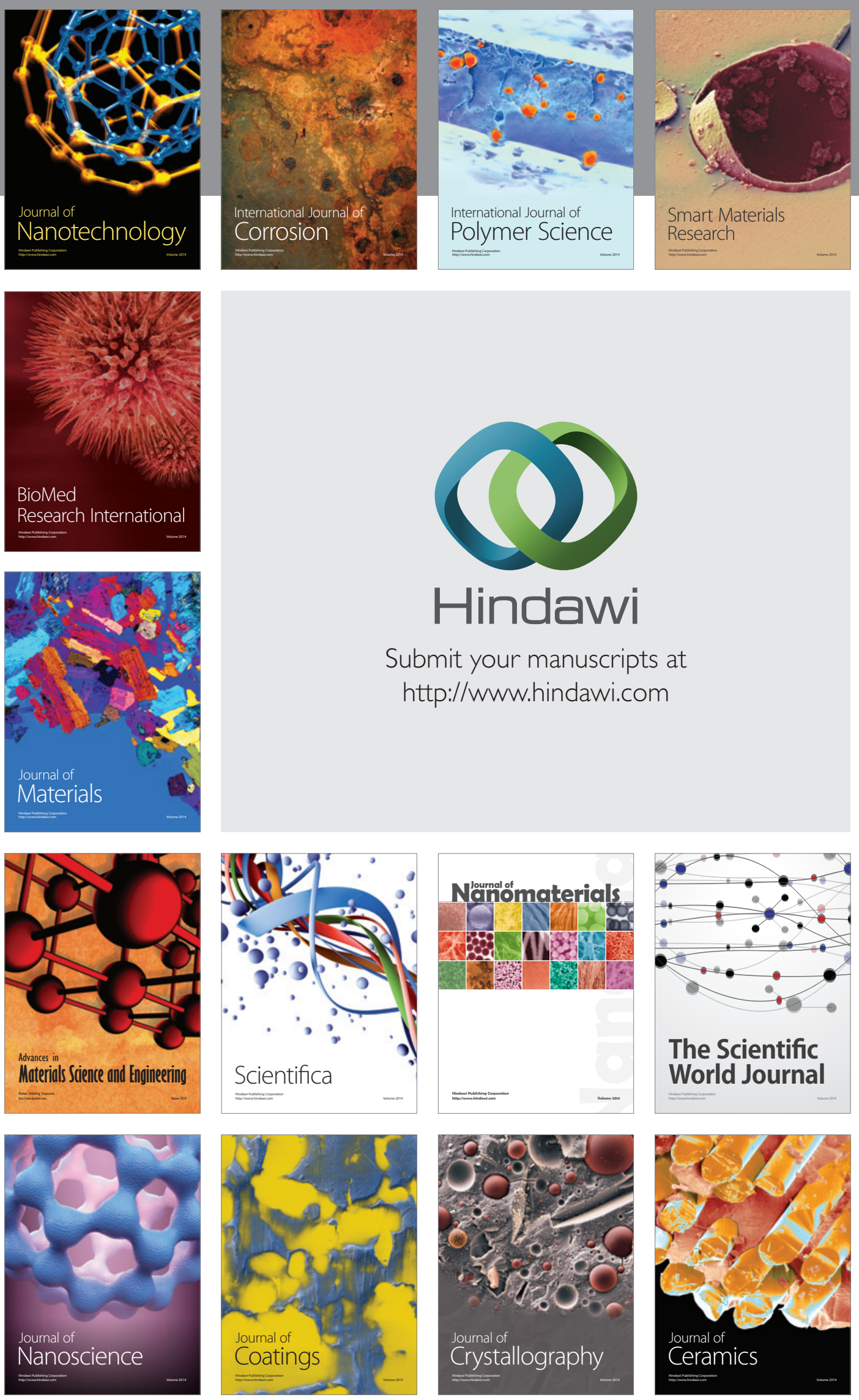

The Scientific World Journal

Submit your manuscripts at

http://www.hindawi.com

\section{World Journal}

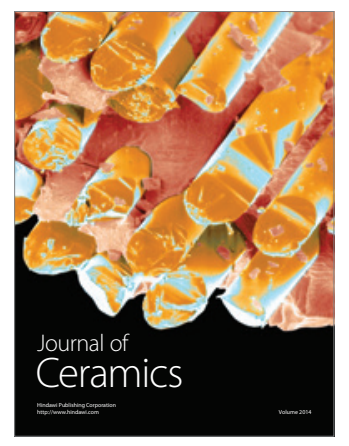

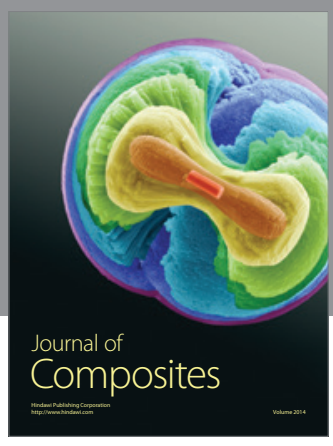
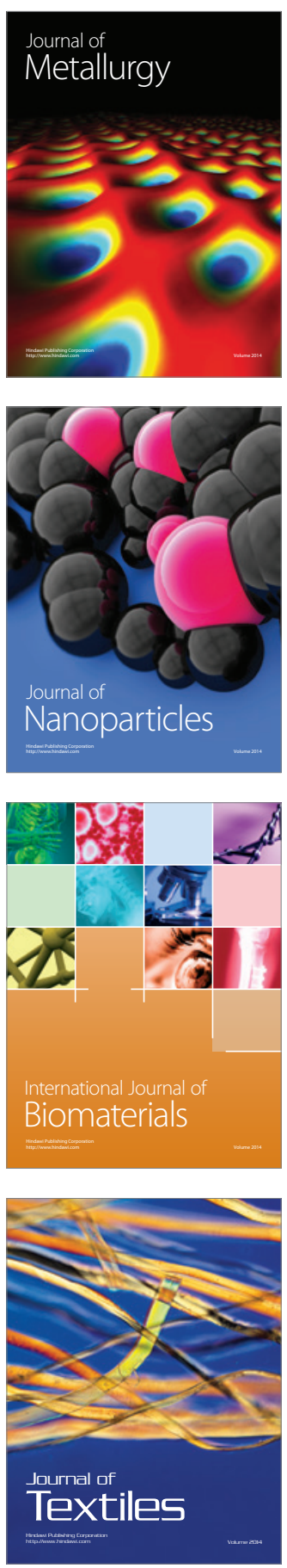